\title{
Increasing Maximum Gain in InAs Quantum Dot Lasers on GaAs and Si
}

\author{
Samuel Shutts ${ }^{1}$, Clemens Spinnler ${ }^{2}$, Zhibo Li ${ }^{1}$, Lydia Jarvis ${ }^{1}$, Emmanuel Le Boulbar ${ }^{1}$, David Hayes ${ }^{1}$ \\ Mingchu Tang ${ }^{3}$, Huiyun Liu ${ }^{3}$ and Peter M. Smowton ${ }^{1}$, \\ ${ }^{1}$ Physics and Astronomy, Cardiff University, Queens Building, The Parade, Cardiff, CF24 3AA. UK \\ ${ }^{2}$ Swiss Nanoscience Institute, University of Basel, Klingelbergstrasse 82, 4056 Basel, Switzerland \\ ${ }^{3}$ Dept of Electronic and Electrical Engineering, University College London, Torrington Place, WC1E TJE, UK.
}

\begin{abstract}
InAs quantum-dot (QD) lasers emitting at $1300 \mathrm{~nm}$ with nominally undoped and modulated p-type doping are studied. Modal-gain measurements indicate a higher gain can be achieved from the ground-state for a given Fermi-level separation with $p$-doping and a reduced temperaturedependence of threshold current for short-cavity lasers.
\end{abstract}

High-performance Si-based InAs/GaAs QD lasers have been demonstrated with CW operation at high temperature $\left(>75^{\circ} \mathrm{C}\right)$ and with long lifetimes, e.g. $10^{4} \mathrm{hrs}$ for broad-area lasers and $10^{6} \mathrm{hrs}$ for ridgewaveguides operating close to room temperature [1][2]. While excellent, these performance characteristics are still insufficient for commercial applications at elevated temperature. Here we describe how laser characteristics can be improved with the use of p-type modulation doping to develop III-V-on-Si technology for high performance lasers and semiconductor optical amplifiers (SOAs) utilised for data-communications applications. The ultimate objectives are to optimise the optical gain per unit length (for high frequency) and to increase operation temperature to the required $125^{\circ} \mathrm{C}$ maximum, while maintaining low drive currents and achieving $\mathrm{CW}$ optical output powers beyond $20 \mathrm{~mW}$. In this work we examine samples grown on GaAs substrates and also similar samples directly grown on silicon substrates using a solid-source molecular beam epitaxy (MBE) system. To realize high-quality III-V lasers on silicon wafers, a nucleation layer made of AlAs was deposited by migration enhanced epitaxy using alternating $\mathrm{Al}$ and $\mathrm{As} 4$ flux at a low growth temperature of $3500^{\circ} \mathrm{C}$ to localise defects at the AIAs/Si interface. Three layers of GaAs were grown at $350{ }^{\circ} \mathrm{C}, 450^{\circ} \mathrm{C}$, and 590 ${ }^{\circ} \mathrm{C}$ for $30 \mathrm{~nm}, 170 \mathrm{~nm}$, and $800 \mathrm{~nm}$, respectively and strained layer superlattices (SLSs) were grown as dislocation filter layers (DFLs) on the top of the GaAs buffer layer. In-situ thermal annealing of the SLS was also carried out further improving the efficacy of filtering defects by increasing the mobility of the defects, leading to defect annihilation before growth of the subsequent layers. 5-layer QD laser structures as represented schematically in Figure 1 are grown either on this template or on GaAs substrates. First we have studied the p-type modulation doping introduced to structures grown on GaAs substrates at levels of $0,3.1$ and 10.5 Be acceptor atoms per dot.

Broad-area lasers and non-lasing test structures (for measuring single-pass modal gain and absorption) were fabricated with as-cleaved facets and devices were operated in pulsed-mode to minimise selfheating. For laser devices, power output versus current characteristics were measured using an integrating sphere. Modulation p-doping can have both positive and negative effects on QD laser performance. For example, the presence of extra holes resulting from the acceptors can have the effect of shifting the position of the quasi-Fermi levels for a given Fermi-level separation making the population of electron and hole dot states more similar. This results in a higher net modal gain and increases the quasi-Fermi level separation at which the peak modal gain shifts from the ground state to the excited state. This type of behaviour can be observed in Figure 2, where the excited state gain occurs at a higher quasi-Fermi level separation for the samples with 3 and 10 acceptors per dot. The presence of acceptors can also lead to enhanced non-radiative recombination and / or optical loss. Measurements of the absorption spectra indicate that the highest doping density samples have the smallest absorption magnitude suggesting that they probably have a smaller dot density than the other samples. It is not yet clear whether this is related to the presence of acceptor ions in the sample or whether it is a coincidence. The threshold current densities $\left(J_{\mathrm{th}}\right)$ for $1,2 \& 3 \mathrm{~mm}$ lasers, of each doping concentration, are plotted in Figure 3 at room temperature and the inset shows the corresponding emission wavelength. The threshold current at this temperature is lower for the 
undoped lasers for all but the $1 \mathrm{~mm}$ long cavities with a p-doping level of 3.1 acceptors per dot. The lasing wavelength blue-shifts with increasing $p$-doping and also with decreasing cavity length (due to the effects of state-filling). Over a temperature range of $200-380 \mathrm{~K}$ no obvious advantage is gained from incorporating p-doping at cavity lengths of 2 and $3 \mathrm{~mm}$ in terms of threshold current versus temperature characteristics. However, as the cavity length is reduced, e.g. for $1 \mathrm{~mm}$ cavities plotted in Figure 4, the temperature dependence becomes slightly less for the 3.1 acceptors per dot, compared with undoped. In summary, we have grown InAs QD lasers on GaAs and directly on Si substrates with different levels of modulation $p$-doping with a view to increasing the maximum modal gain and improving the high temperature performance. Measurements of the net modal gain on GaAs substrates indicate that a modest increase in the maximum gain obtained from the ground state is achieved for a given Fermi-level separation with increasing levels of p-modulation doping. However, this does not necessarily translate to improved laser performance due to unintentional changes in the dot density and the degree of inhomogeneous broadening achieved in the samples with p-modulation doping and also due to some increase in the level of non-radiative recombination in these samples.
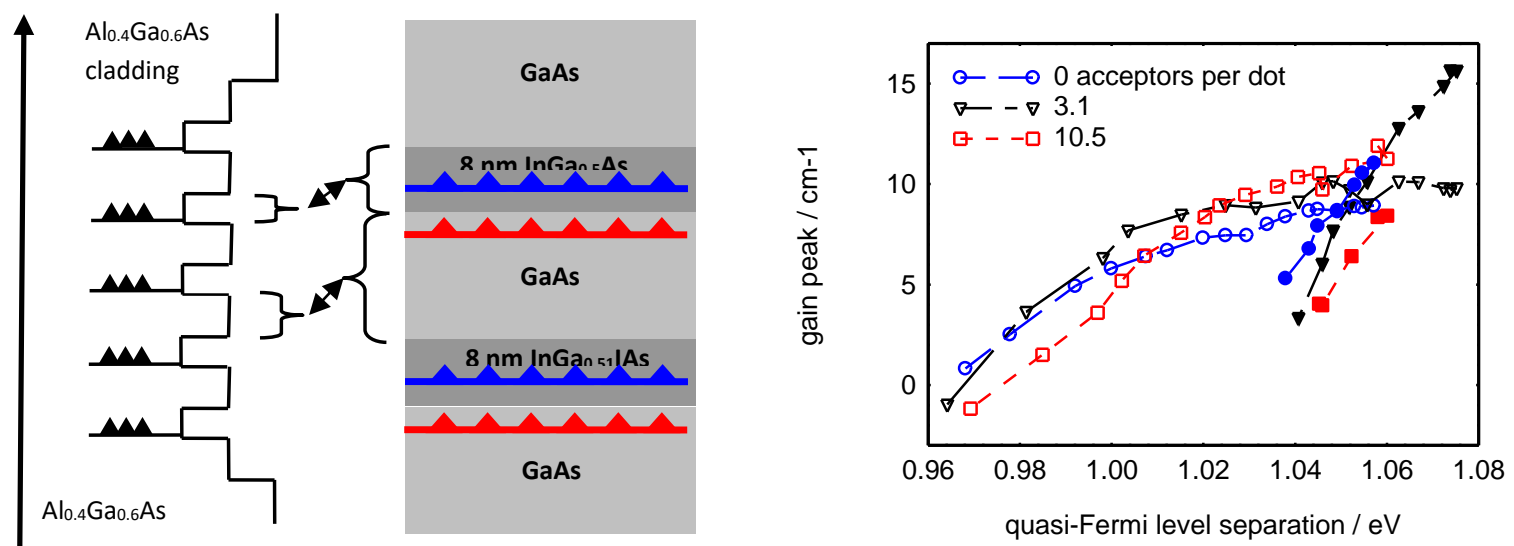

Figure 1: Schema of layer structure for InAs dot lasers Figure 2: Peak net gain versus transparency point (quasiindicating p-doped layers grown below in GaAs immediately Fermi level separation) for samples of 0, 3.1 and 10.5 below InGaAs well.

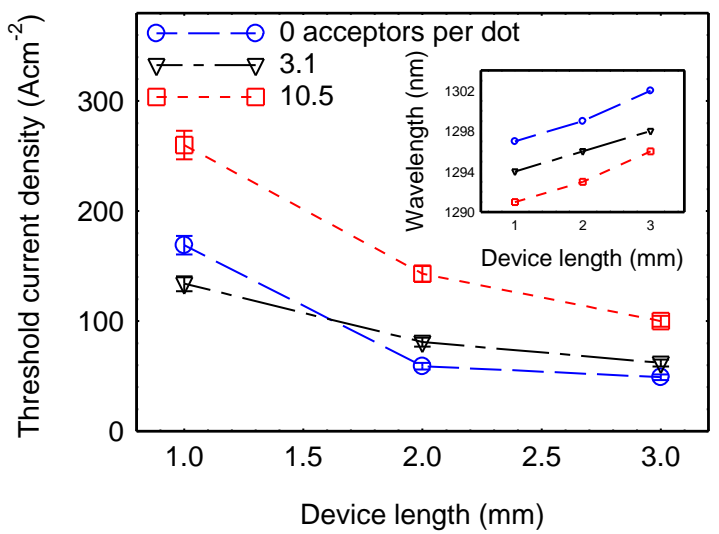
acceptors per dot. Open/closed symbols for ground/excited state

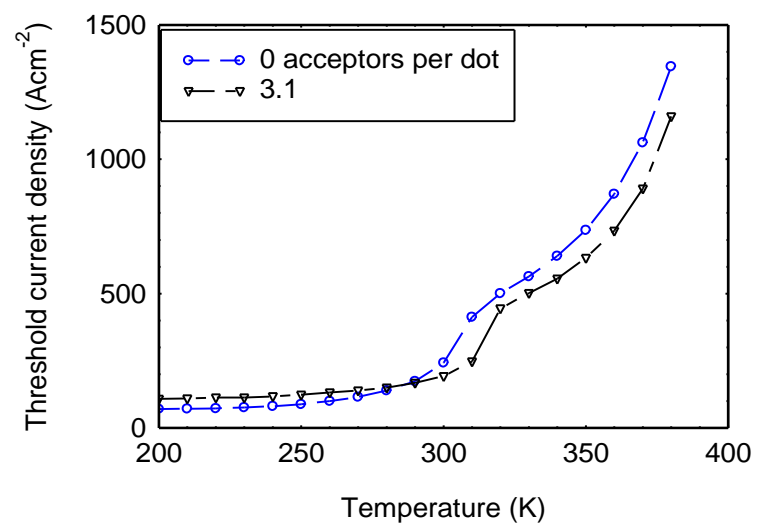

Figure 3: Room temperature threshold current density for $2 \mathrm{~mm}$ long lasers containing 0, 3 and 10 acceptor atoms per dot. Inset shows emission wavelength.

Figure 4: Threshold current density vs. temperature of $1 \mathrm{~mm}$ long cavities for 0 and 3.1 acceptors per dot.

This work was supported by EPSRC grant EP/P006973/

[1] S. Chen et al., "Electrically pumped continuous-wave III-V quantum dot lasers on silicon," Nat. Photonics, vol. 10, no. 5, pp. 307-311, 2016.

[2] D. Jung et al., "Impact of threading dislocation density on the lifetime of InAs quantum dot lasers on Si," Appl. Phys. Lett., vol. 112, no. 15, p. 153507, 2018. 